\title{
A spectrum efficient node clustering method for WMSN
}

\author{
Zheyuan Xiong ${ }^{a}$, Xiaomin Xiong ${ }^{b}$ \\ ${ }^{1}$ Department of Security Technology, Jiangxi Police Institute, Nanchang, 330103, China \\ aemail: xiongzhey@126.com, bemail:xxm913@sohu.com
}

Keywords: spectrum efficiency; field of view; Clustering; wireless multimedia sensor networks

\begin{abstract}
In wireless multimedia sensor networks (WMSN), visual correlation exists among multiple neighboring sensors. Overlapping Field of View in dense networks leads to considerable redundancy in the collected images. A spectrum efficient clustering mechanism is proposed for efficiently gathering images in WMSN. The main objective of the proposed clustering method is spectrum utilization improving and network lifetime prolongation. This aim is achieved by finding the optimal locations for cluster heads, which operate on different channels for concurrently collecting images from adjacent cameras, such that the number of channels required for frequency reuse is minimized. Simulations show that the proposed clustering algorithm can effectively enhance the network throughput and the energy efficiency of camera sensors.
\end{abstract}

\section{Introduction}

WMSN contain video sensors, microphones and other sensors producing multimedia content. Sensor devices are constrained in terms of battery, memory, processing capability, and achievable data rate. Hence, efficient use of these scarce resources is mandatory. WMSN promise a wide range of potential applications such as visual surveillance, law enforcement reports, traffic control systems, advanced health care delivery, and industrial process control [1,2].

For WMSN, the sensing region of the nodes is different from the traditional nodes used in WSN. The multimedia node has a defined field of view (FoV) and can capture objects within that region only [3]. These overlapped FoV further incur a certain degree of visual correlation among multiple cameras, thus leading to unnecessary redundancy in the captured images, as shown in Fig. 1. Camera sensors can perform collaborative coding to remove such redundancy. This collaborative coding rate depends on the degree of the correlation between the two cameras [4]. To increase network throughput of a WMSN, we proposed a scalable network architecture that overcomes the limitations of energy and spectrum.

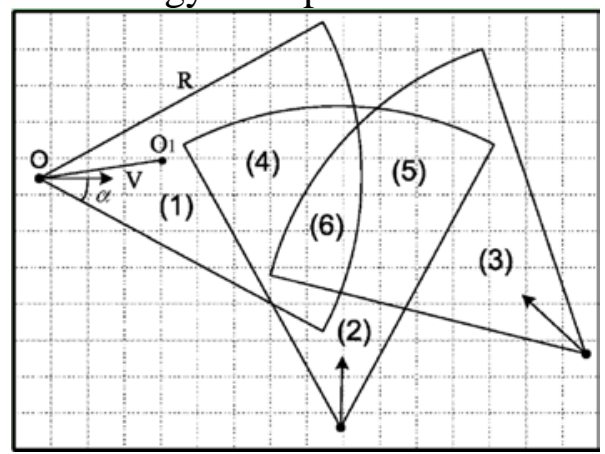

Fig. 1 Field of views of multiple cameras

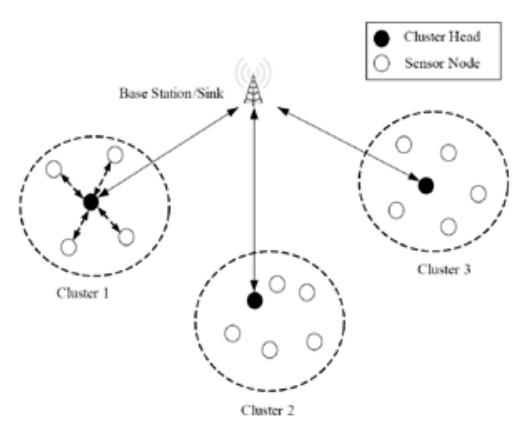

Fig. 2 Clustering of sensor nodes in WMSN

The clustering is a standard approach for achieving efficient and scalable control in a large scale network, as shown in Fig. 2. Nodes communicate their data over shorter distances to their respective cluster head $(\mathrm{CH})$. The cluster head aggregates these data, and communicates with their neighboring cluster heads and sink/base station [5]. We argue that this concentration can lead to a variance in energy consumption among sensor nodes and is able to cause rapid partition of the network. Therefore, to prolong the lifetime of a WMSN, placing $\mathrm{CH}$ at proper locations that facilitate frequency reuse is of significant importance. In this way, a load balancing among nodes for data 
dissemination is achieved.

Because of the difference between radio neighborhood and sensed region by the multimedia node, the coverage techniques and node clustering in WSN do not satisfy WMSN requirements. After $\mathrm{CH}$ is located, we took the idea for making clusters based on overlapping FoV of the camera and accordingly evaluating intersection polynomial of FoV of two nodes and computing the overlapped area are the keys to figure out the clusters and cluster membership.

\section{Related Works}

The nodes are often grouped together into disjoint and mostly non-overlapping groups are called clusters. Clusters are used to minimize communication latency and improve energy efficiency. Leader of every cluster is often called the cluster head $(\mathrm{CH})$ and generally has to perform more functions as compared to normal sensor node [6].

The typical clustering routings protocols in WSNs include Low-energy Adaptive Clustering Hierarchy (LEACH), Hybrid Energy-Efficient Distributed clustering (HEED), Distributed Weight-based Energy-efficient Hierarchical Clustering protocol (DWEHC), Position-based Aggregator Node Election protocol (PANEL), Two-Level Hierarchy LEACH (TL-LEACH), Unequal Clustering Size (UCS) model, Energy Efficient Clustering Scheme (EECS), Energy-Efficient Uneven Clustering (EEUC) algorithm, Algorithm for Cluster Establishment (ACE), Base-Station Controlled Dynamic Clustering Protocol (BCDCP), Power-Efficient Gathering in Sensor Information Systems (PEGASIS), Threshold sensitive Energy Efficient sensor Network protocol (TEEN), The Adaptive Threshold sensitive Energy Efficient sensor Network protocol (APTEEN), Two-Tier Data Dissemination (TTDD), Concentric Clustering Scheme (CCS), Hierarchical Geographic Multicast Routing (HGMR), and etc [7]. Clustering routing is becoming an active branch of routing technology in WSNs on account of a variety of advantages, such as more scalability, data aggregation/fusion, less load, less energy consumption, more robustness, etc [8].

We assume wireless sensor nodes with fixed lenses providing a $\theta$ angle $\mathrm{FoV}$, densely deployed in a random manner. The assumption of fixed lenses is based on the current WMSN platforms. Almost all of them have fixed lenses and only high powered PTZ cameras have movement capabilities.

\section{Cluster Head Location}

Node clustering is performed to provide distributed control over data transmission. Lacking any traffic control on the multimedia sources can quickly hamper the performance of nodes if the traffic exceeds the capacity of forwarding links and routes. In a cluster-based configuration, a cluster head controls the transmission of nodes participating as members and therefore avoids awkward performance degradation with an increased number of traffic sources [9].

To facilitate frequency reuse, the neighboring clusters must be assigned with different channels and the cameras must be able to operate on the channels of their associated clusters. Since the maximum distance between two neighboring clusters is 2-hop distance, by graph coloring theorems, this implies that the maximum degree of hubs should be less than the available orthogonal channels to ensure the effectiveness of frequency reuse. For this purpose, we define the optimal cluster head location problem as follows.

Consider WMSN modeled by a graph $G=(C, L)$, where $C$ is a set of cameras, i.e., $C=\left\{c_{1}, c_{2}, \ldots c_{n}\right\}$, and $L$ is a set of links. If $c_{i}$ and $c_{j}$ are within 1-hop range of each other, a link $\left(c_{i}, c_{j}\right)$ exists. The 1-hop neighborhood of $c_{i}$, denoted by $S_{i}^{1}$, is a set consisting of $c_{i}$ and cameras within 1-hop range of ${ }^{c_{i}}$. The 2-hop neighborhood of $c_{i}$, denoted by $S_{i}^{2}$, is a set of cameras within 2-hop range of $\mathrm{c}_{i}$, excluding $\mathrm{c}_{i}$.

The degree of a $\mathrm{CH} \mathrm{h}$, denoted by $\operatorname{deg}(\mathrm{h})$, is the total number of $\mathrm{CHs}$ (except $\mathrm{h}$ ) that reside 
within the 2-hop range of the $\mathrm{CH} h$. Given a graph $\mathrm{G}=(\mathrm{C}, \mathrm{L})$ and a set of potential $\mathrm{CH}$ locations $\mathrm{F}=\mathrm{C}$, find a subset $\mathrm{F}^{\prime} \subseteq \mathrm{F}$ such that the maximum degree of $\mathrm{CHs}$, $\max _{\mathrm{h} \in \mathrm{F}},(\operatorname{deg}(\mathrm{h}))$, is minimum, and for all $c_{i} \in C$, there is at least one $\mathrm{CH} h \in \mathrm{F}^{\prime}$ for which $\left(h, c_{i}\right) \in L$. The set of CHs $\mathrm{F}^{\prime}$ is actually a dominating set of the WMSN so that every camera sensor is at most 1-hop away from at least one of the $\mathrm{CHs}$ in $\mathrm{F}^{\prime}$.

We assign a variable $\mathrm{X}_{\mathrm{i}}$ for each camera sensor $\mathrm{c} \in \mathrm{C}$, which is allowed $0 / 1$ values. This variable will be set to 1 if a $\mathrm{CH}$ is placed at the location of ${ }^{\mathrm{C}_{i}}$. Consequently, the optimal cluster head location problem can be formulated as an integer nonlinear program.

$$
\begin{aligned}
& \text { Min } \quad y \\
& \text { s.t } \sum_{j: c_{i} \in S_{i}^{1}} x_{j} \geq 1, \quad \forall c_{i} \in C \\
& \sum_{j: c_{i} \in S_{i}^{2}} x_{i} x_{j} \leq y, \quad \forall c_{i} \in C \\
& x_{j} \in\{0,1\}, \quad \forall c_{j} \in C
\end{aligned}
$$

The objective function $y$ is the maximum degree of all CHs $\left(\left\{c_{i} \mid x_{i}=1\right\}\right)$. The first constraint states that each camera must reside within the 1-hop neighborhood of at least one $\mathrm{CH}$, whereas the second constraint indicates that the degree of each $\mathrm{CH}$ must be less than the maximum value. As the second constraint (3) is quadratic, the formulated integer program is not linear. To linearize integer nonlinear program, the quadratic constraint (3) is eliminated by applying the techniques proposed in [10]. More specifically, the product $x_{i} x_{j}$ is replaced by a new binary variable ${ }^{\mathrm{w}_{\mathrm{ij}}}$, on which several additional constraints are imposed. As a consequence, we can reformulate the integer nonlinear program exactly to an integer linear Program by introducing the following linearization constraints:

$$
\begin{aligned}
& \sum_{j: v_{i} \in S_{i}^{2}} \mathrm{w}_{i j} \leq \mathrm{y}, \quad \forall \mathrm{v}_{\mathrm{i}} \in \mathrm{V} \\
& \mathrm{w}_{\mathrm{ij}} \leq \mathrm{x}_{\mathrm{i}}, \mathrm{w}_{\mathrm{ij}} \leq \mathrm{x}_{\mathrm{j}}, \quad \forall \mathrm{v}_{\mathrm{i}}, \mathrm{v}_{\mathrm{j}} \in \mathrm{V} \\
& \mathrm{w}_{\mathrm{i} j} \geq \mathrm{x}_{\mathrm{i}}+\mathrm{x}_{\mathrm{j}}-1, \quad \forall \mathrm{v}_{\mathrm{i}}, \mathrm{v}_{\mathrm{j}} \in \mathrm{V} \\
& \mathrm{w}_{\mathrm{ij}} \geq 0, \quad \forall \mathrm{v}_{\mathrm{i}}, \mathrm{v}_{\mathrm{j}} \in \mathrm{V}
\end{aligned}
$$

and removing the quadratic constraint (3). By relaxing variables $x_{i} \in\{0,1\}$ to $x_{i} \in[0,1]$, we get the relaxed linear program consisting of the objective function (1) along with constraints (2), (5), (6), (7), (8), and $x_{j} \geq 0, \forall v_{j} \in V$.

Given an instance of optimal cluster head location problem modeled by the integer program, an approximation algorithm is proposed as following:

First solve the relaxed linear program to get an optimal fractional solution, denoted by $\left(x^{\prime}, y^{\prime}\right)$, where $\mathrm{x}^{\prime}=\left\{\mathrm{x}_{1}{ }_{1}, \mathrm{x}_{2}{ }_{2}, \ldots, \mathrm{x}^{\prime}{ }_{|c|}\right\}$, and round $\mathrm{x}^{\prime}{ }_{1}$ to integers $\overline{\mathrm{x}}_{\mathrm{i}}$ by a random rounding procedure. This procedure consists of three steps:

(1) first set all $\bar{x}_{i}$ to be 0 ; (2) then let $\bar{x}_{i}=1$ with probability ${ }^{x^{\prime}}{ }_{i}$ and execute this step for $\log (\mathrm{n})+2$ times, where $\mathrm{n}$ is the number of sensor nodes in the network. 
Step (2) yields an integer solution $(\overline{\mathrm{x}}, \overline{\mathrm{y}})$, where vector $\overline{\mathrm{x}}=\left\{\overline{\mathrm{x}}_{1}, \overline{\mathrm{x}}_{2}, \ldots, \overline{\mathrm{x}}_{|\mathrm{V}|}\right\}$. To ensure $(\bar{x}, \bar{y})$ is a feasible solution to optimal cluster head location problem, step (2) is repeated until camera is the neighbor of at least on $\mathrm{CH}$, and the maximum degree $\overline{\mathrm{y}}$ satisfies the condition that $\bar{y} \leq \delta \alpha^{2} y$, where $\delta=\left(1-e^{-\alpha \theta}\right)^{-1}, \alpha=\log (n)+2$ and $\theta=\min _{x^{\prime}{ }_{i} \neq 0 \wedge i \leq n} x{ }^{\prime}$ are some constants.

The last step (3) is to further reduce the maximum hub degree by removing the possible redundant $\mathrm{CH}$, which has its one hop neighbors (including itself) covered by other CHs.

\section{Member Camera Assignment}

Now, let us consider the set $\mathrm{S}=\{\mathrm{S} 1, \mathrm{~S} 2, \ldots, \mathrm{SN}\}$ of wireless camera nodes belonging to the same tier of a network randomly deployed. The cluster formation algorithm is executed in a centralized manner by the sink after deploying the network.

(1)Bootstrap

At node bootstrap, each sensor $\left\{\mathrm{S}_{\mathrm{i}}, \mathrm{i}=1, \ldots, \mathrm{N}\right\}$ transmits its position $\left(\mathrm{x}_{\mathrm{i}}, \mathrm{y}_{\mathrm{i}}\right)$ and orientation $\alpha_{i}$ to the sink. To accomplish this step any efficient sensor routing algorithm can be used. Thus, the clustering algorithm is not bound to how the sink receives this information. If there is an un-connected node in the network, it cannot announce itself and thus will not be considered in the algorithm.

(2)Cluster Formation

(i) Initially, the sink creates an empty cluster associated with an un-clustered multimedia node of S. Thus, that node will be clustered as the first member (i.e., cluster-head) of the established cluster.

(ii) Then, the sink finds the qualified un-clustered nodes for joining to that first member by computing the area of overlapped polygons of their FoV. From position and orientation of nodes, the sink computes the overlapped polygon area $\left(D_{i j}\right)$ between each un-clustered multimedia node and the first member of the established cluster as discussed in Section 3. If the computed overlapped area is equal or greater than the area determined by the overlapping scale $(\gamma)$, the un-clustered node will be clustered as a member of the established cluster.

(iii) When no more nodes can be added to the cluster, the sink takes a new un-clustered node, begins a new cluster and goes to step (ii).

(3)Membership notification

We assume that the sink uses any energy-efficient sensor routing algorithm to notify to each first-member of every cluster about its cluster-ID and what are the members of the cluster. Then, each first-member sends a packet to the members of his cluster notifying them about the cluster which they belong to.

The algorithm is executed by the sink once upon deployment and thus all nodes will become clustered. If a node joins to the network hereinafter, it has to send its position and orientation to the sink for announcing itself as a new node. The sink computes the FoV of the new node and finds the first cluster that can accept it as a new member. For this purpose, the sink computes the overlapping regions between FoV of the new node and the first-member of each cluster and checks whether he is satisfying the cluster membership test [11].

Then, the sink sends a message to the first-member in order that this node re-organizes the cluster with the new member. Depending on the application, this notification may suppose a new reconfiguration in the monitoring task. On the other hand, each node periodically sends a Hello message to the first-member notifying that he is alive. When a node dies, the first-member will notify the rest of the members about the new cluster set and will reconfigure any parameter related to the cluster. The first-member also periodically notifies to his cluster members about his availability. If a first-member dies, the cluster members will notify to the sink their availability to 
belong to another cluster or to create a new cluster. Note that the beaconing among cluster members implies low overhead since cluster sizes have few nodes and hello periods can be on the order of duty-cycle sensing periods.

\section{Simulation and Analysis}

In this section, we evaluate the performance of the proposed clustering algorithm. We study the efficiency of the proposed network deployment approach that consists of cluster head selection and camera assignment algorithms. For all the experiments conducted in this paper, the sensor field is of $160 \times 160 \mathrm{~m}^{2}$ with a varied number of nodes ranging from 100 to 500 . For distributed DCT-based image compression, we used standard gray scales Lena, Cameraman, and Peppers are used for distributed DCT-based compression experiments.

Table 1 show the energy gain obtained in HEED and our algorithm with respect to PEGASIS with sufficient energy. It allows energy saving in both HEED and our algorithm where they at the most consume $45 \%$ of what PEGASIS consumes for 100-node network. When increasing the network size, the gain is much improved mainly using our algorithm. This is because in our algorithm more data packets are delivered than in HEED.

Table 1 Energy ratio per correctly received byte

\begin{tabular}{|c|c|c|}
\hline $\begin{array}{l}\text { Network } \\
\text { size }\end{array}$ & $\begin{array}{l}\text { Energy per correctly } \\
\text { received byte ratio of } \\
\text { our algorithm }\end{array}$ & $\begin{array}{l}\text { Energy per correctly received } \\
\text { byte ratio of HEED }\end{array}$ \\
\hline 100 & 0.45 & 0.45 \\
\hline 200 & 0.28 & 0.3 \\
\hline 300 & 0.26 & 0.27 \\
\hline 400 & 0.2 & 0.23 \\
\hline 500 & 0.16 & 0.18 \\
\hline
\end{tabular}

Prolonging the network lifetime is our primary goal when proposing our algorithm. We conduct experiments with nodes assigned insufficient energy to capture network lifetime. We use the duration of the video received by the sink as a lifetime metric. Table 2 plots the network lifetime for the three protocols with insufficient energy as the network size increases. The network lifetime slightly decreases with the number of nodes increasing since a larger number of nodes results in a higher number of floods and hence bigger amount of energy is consumed in the network. Our algorithm, however, achieves better performances compared to the two others.

Table 2 Lifetime with insufficient energy (seconds)

\begin{tabular}{|c|c|c|c|}
\hline Network size & Lifetime of our algorithm & Lifetime of HEED & Lifetime of PEGASIS \\
\hline 100 & 18.5 & 12.5 & 8.5 \\
\hline 200 & 19 & 13 & 8 \\
\hline 300 & 18.5 & 12 & 7.5 \\
\hline 400 & 18.4 & 11.5 & 6 \\
\hline 500 & 17.5 & 12 & 5.2 \\
\hline
\end{tabular}

Regarding video quality, our algorithm outperforms both HEED and PEGASIS mainly for insufficient energy scenarios as shown in Table 3. Video quality decreases with the network size increasing since larger number of nodes results in a higher number of floods and hence bigger amount of losses which affect directly the quality of received video.

Table 3 Overall PSNR with insufficient energy

\begin{tabular}{|c|c|c|c|}
\hline Network size & PSNR of our algorithm & PSNR of HEED & PSNR of PEGASIS \\
\hline 100 & 27 & 22.5 & 15 \\
\hline 200 & 26 & 22 & 16 \\
\hline 300 & 25 & 19 & 14.5 \\
\hline 400 & 23 & 18.5 & 14 \\
\hline 500 & 22 & 17.5 & 14.5 \\
\hline
\end{tabular}




\section{Conclusion}

Habitat and process monitoring applications have resource and energy utilization requirements in WMSN. To improve spectrum utilization and prolong the network lifetime and hence provide useful applications, WMSN require scalable network architecture. In this paper, a spectrum efficient clustering method for WMSN is proposed. The optimal hub locations are found such that the required number of orthogonal channels for frequency reuse is minimum. Cluster-membership is decided based on overlapping FoV areas. Simulations show that the proposed algorithm is effective and efficient in improving the spectrum utilization and energy efficiency in a WMSN.

\section{Acknowledgement}

This work was supported by Science and Technology Project of Jiangxi Department of Education (GJJ14772), Application Innovation Projects of the Ministry of Public Security (2013YYCXJXST045), Science and Technology Landing Project of Jiangxi University (KJLD13098).

\section{References}

[1] I. F. Akyildiz, T.Melodia, and K.R. Chowdhury, A survey on wireless multimedia sensor networks, Computer Networks, 2007, 51(4): 921-960.

[2] S. Ehsan and B. Hamdaoui, A survey on energy-efficient routing techniques with QoS assurances for wireless multimedia sensor networks, IEEE Communications Surveys and Tutorials, 2012, 14(4): 256-278.

[3] A. Newell and K. Akkaya, Self-Actuation of Camera Sensors for Redundant Data Elimination in Wireless Multimedia Sensor Networks, IEEE International Conference on Communication, 14-15 June 2009, pp. 1-5.

[4] R. Dai and I. F. Akyildiz, A spatial correlation model for visual information in wireless multimedia sensor networks, IEEE Transactions on Multimedia, 2009, 11(6): 1148-1159.

[5] B.Dieber, C. Micheloni, and B. Rinner, Resource-aware coverage and task assignment in visual sensor networks, IEEE Transactions on Circuits System Video Technology, 2011, 21(10): 1424-1437.

[6] M Alaei, JM Barcelo-Ordinas, A Method for Clustering and Cooperation in Wireless Multimedia Sensor Networks, Sensors, 2010, 10(4): 3145-3169.

[7] Xuxun Liu, A Survey on Clustering Routing Protocols in Wireless Sensor Networks, Sensors, 2012, 12(8): 11113-11153.

[8] H. Zeghilet, M. Maimour, N. Badache, F. Lepage, On the use of passive clustering in wireless video sensor networks, International Journal of Sensor Networks, 2012, 11(2): 67-80.

[9] D Gupta, SL Aswal, SK Chaurasiya, Effect of Obstacles in the formation of FoV based Clusters in Wireless Multimedia Sensor Network, International Journal on Computer Science \& Engineering,2012, 4(8): 1427-1434.

[10] P Kumar, N Chand, Clustering in Wireless Multimedia Sensor Networks, Journal of Sensor Technology, 2013, 03(4): 126-132.

[11] GA Shah, F Alagoz, EA Fadel, OB Akan, A Spectrum-Aware Clustering for Efficient Multimedia Routing in Cognitive Radio Sensor Networks, IEEE Transactions on Vehicular Technology, 2014, 63(7): 3369-3380. 